which can then be subjected to extensive analysis, usually impossible on large molecules directly.

The 'RNA world' hypothesis is gaining credibility as more RNA-governed activities are revealed. The discovery that peptidyl transferase activity might be an RNA-mediated reaction was unexpected; now decoding is joining the RNA 'club'. Are we soon going to be surprised by finding a ribosomal protein that has an essential function? Today's ribosome evolved to function with rRNAs, proteins and translation factors acting together to ensure translational efficiency and accuracy. Painstaking investigation of the ribosome's different functions will help us understand the complex and subtle interplay between the components of the translational apparatus.

Renée Schroeder is in the Institute of Microbiology and Genetics, University of Vienna, Dr Bohrgasse 9, A-1030 Vienna, Austria.

\title{
The search for symmetry
}

\section{VictorJ. Emery}

AT a conference on superconductivity held in July*, the liveliest discussion centred on the symmetry of the ordered state of high-temperature superconductors so lively in fact that the debate spilled out into the corridors, and an impromptu session had to be organized to give everyone a chance to air their views. This apparently esoteric property is important for what it may reveal about the nature of high-temperature superconductivity, which has sparked so much effort around the world since the initial discovery in 1986. Knowledge of the symmetry in question would not unequivocally identify the underlying mechanism of hightemperature superconductivity but it could serve to narrow the field.

The simplest way to think about the problem is to focus on the copper oxide planes in which the primary superconducting charges reside, and to imagine that the copper ions form a square lattice. There are good reasons to believe that the superconducting state of the cuprates, like that of conventional superconductors, is composed of pairs of electrons with even angular momentum. For a square lattice the allowed quantized even angular momenta are zero ( $s$-wave) and two ( $d$ wave). Typically a short-range attractive interaction gives rise to $s$-wave pairing but a short-range repulsion combined with a longer-range attraction may favour a higher angular momentum state, which serves to keep the colliding particles away from each other and optimize the attraction. This is the essence of the argument that led to suggestions of $d$-wave pairing in liquid ${ }^{3} \mathrm{He}$ in 1960.

From this perspective, the nature of the pairing state helps us to identify the form of the effective interaction between the conduction electrons. But that is not the whole story: collective effects such as spin fluctuations may come into play and either

*Fourth International Conference on Materials and Mechanisms of Superconductivity/High-Temperature Superconductors, Grenoble, France, 5-9 July 1994. modify or completely determine the nature of the pairing state. In the case of liquid ${ }^{3} \mathrm{He}$, ferromagnetic spin fluctuations lead to $p$-wave pairing, with odd angular momentum. About ten years ago, it was suggested that the exchange of antiferromagnetic spin fluctuations might be the source of superconductivity in organic and heavy fermion materials, and it was naturally one of the many mechanisms considered in the first frantic days of high-temperature superconductivity, especially after the discovery that the 'parent' insulating compounds are antiferromagnetically ordered.

Since then the idea has been pursued vigorously by several groups around the world. This is the mechanism that raises the stakes in the search for symmetry, for, at least in its simplest form, it is ruled out if the pairing is $s$-wave. Antiferromagnetic spin fluctuations carry one unit of spin and, as they are emitted or absorbed, they effectively flip the spin of a conduction electron. For singlet pairs, the spin flip brings along a sign change which converts an otherwise attractive exchange force into a repulsion. The saving grace is that the interaction follows the spatial oscillations of the spin configurations, so the pairs may arrange their angular momentum to catch the oscillations on the rise and experience an effective attraction. The lowest, and most effective, angular momentum state of this kind is $d$-wave.

What does experiment have to say? Much hard work, both theoretical and experimental, has gone into this question. For the most thoroughly studied material, $\mathrm{YBa}_{2} \mathrm{Cu}_{3} \mathrm{O}_{7-\delta}$, there are clear indications that the energy gap is anisotropic and that it is zero at some places on the Fermi surface. But that can be true of either $s$-wave or $d$-wave. The experiments which are stirring so much interest in the community have addressed the question more directly by asking if the average value of the energy gap vanishes, or alternatively by looking for evidence that sign of the energy gap is changed by a $90^{\circ}$ rotation, both of which would be symptoms of $d$-wave pairing.

At this point the message is mixed. If we take the existing experiments at face value and thereby sidestep much of the current debate, it appears that the energy gap has both $d$-wave $\mathrm{w}^{1-3}$ and $s$-wave $\mathrm{w}^{4-6}$ components.

On closer examination, this should not be surprising. Most discussions have proceeded on the assumption that, although $\mathrm{YBa}_{2} \mathrm{Cu}_{3} \mathrm{O}_{7-\delta}$ is an orthorhombic material, the simple square lattice analysis is not too bad. But this assumption is questionable: in $\mathrm{YBa}_{2} \mathrm{Cu}_{3} \mathrm{O}_{7-\delta}$ there is a significant electronic anisotropy in the form of a set of conducting $\mathrm{CuO}$ chains running along one axis of the copper oxide planes. The chains may participate in the superconductivity through their proximity to the $\mathrm{CuO}_{2}$ planes and, in turn, impose an anisotropic potential onto the planes themselves. Strictly speaking, the different angular momentum states must be mixed, and there is no reason to believe that the mixing is insignificant. Indeed the superfluid density is quite different along the directions parallel to and perpendicular to the chains. All of this leaves us with the much more difficult task of deciding which is the dominant angular momentum state, and that requires a much more detailed theoretical analysis than has been carried out so far. A similar argument may be made for $\mathrm{Bi}_{2} \mathrm{SrCaCu}_{2} \mathrm{O}_{8+x}$, which is anisotropic because of a lattice modulation that is known to influence the electronic properties, although to a smaller degree than the chains in $\mathrm{YBa}_{2} \mathrm{Cu}_{3} \mathrm{O}_{7-\delta}$.

Obviously it would be cleaner to carry out the same analysis on tetragonal materials, for which the different angular momenta are not mixed. The problem appears to be that available $\mathrm{YBa}_{2} \mathrm{Cu}_{3} \mathrm{O}_{7-\delta}$ samples have been of much higher quality than their tetragonal cousins. Nevertheless, point contact tunnelling ${ }^{7}$ indicates that $\mathrm{HgBa}_{2} \mathrm{CuO}_{4+\delta}$ is $s$-wave, and there is convincing evidence 8 of $s$-wave pairing in the electron-doped material $\mathrm{Nd}_{2-x} \mathrm{Ce}_{x} \mathrm{CuO}_{4}$, which also shows striking antiferromagnetic behaviour. No one will be satisified by such limited information, but experiments on tetragonal materials may well be the wave of the future.

Victor J. Emery is in the Department of Physics, Building 510, Brookhaven National Laboratory, Upton, New York 11973, USA.

\footnotetext{
1. Wollman, D. et al. Phys. Rev. Lett. 71, 2134 (1993).

2. Tsuei, C. etal. Phys. Rev. Lett. 73, 593 (1994).

3. Mathai, A., Gim, Y., Black, R. C., Amar, A. \& Wellstood, F. C., Univ. of Maryland preprint (1994).

4. Chaudhari, P. \& Lin, S. Y. Phys. Rev. Lett. 72, 1084 (1994).

5. Sun, A. G. etal. Phys. Rev. Lett. 72, 2267 (1994)

6. Buan, J. etal. Phys. Rev. Lett. 72, 2632 (1994)

7. Chen, J. etal. Phys. Rev. B49, 3683 (1994).

8. Wu, D. H. etal. Phys. Rev. Lett. 70, 85 (1993).
} 